\title{
Using Gelfoam Technique to Reduce the Incidence of Seroma Formation after Modified Radical Mastectomy
}

\author{
Tamer Mohamed Elshahidy*, Mohamed Mahmoud Mokhtar, Mahmoud Abdou Yassin
}

Department of General Surgery, Faculty of Medicine, Zagazig University, Egypt.

*Corresponding author: Tamer Mohamed Elshahidy, E-mail: shahidytamer@yahoo.com

\begin{abstract}
Background: Breast cancer is the most prevalent cancer in our surgical practice in Egypt. Seroma is one of the most annoying complications after modified radical mastectomy and axillary clearance. Different modalities have been used to decrease the incidence of seroma.

Objective: To assess the impact of using gelfoam in decreasing seroma formation following modified radical mastectomy.

Patients and methods: This randomized controlled trial was done on eighty patients operated upon for modified radical mastectomy between January 2019 and November 2020 in Zagazig University Hospitals. Patients were randomly allocated into two groups; in each one forty patients. In the first group (group A) we used the gelfoam under the mastectomy and axillary flaps; in the second group (group B) we did the same surgical technique without using the gelfoam.
\end{abstract}

Results: We noticed that the incidence of seroma formation among group (A) was $2.5 \%$ versus $17.5 \%$ in group (B), with statistically significant difference between both of them $(\mathrm{P}<0.05)$.

Conclusion: The use of gelfoam is an effective procedure which reduces the incidence of seroma formation under mastectomy flaps.

Keywords: Gelfoam, Modified radical mastectomy, Seroma.

\section{INTRODUCTION}

Breast cancer is one of the most common cancers in Egypt and represents $18.9 \%$ of all cancers (32.04 percent in women and 2.2 in men) ${ }^{(\mathbf{1})}$. The most common surgical operation for this cancer is modified radical mastectomy (2). Many problems commonly occur after this procedure such as skin flaps necrosis, hematoma, seroma and surgical site infection.

Seroma is a clinically apparent serous fluid accumulation at the surgical site ${ }^{(2)}$. It is considered one of the most common complications that may postpone starting adjuvant therapy causing considerable patient distress associated with increasing the risk of surgical site infection ${ }^{(3)}$. The frequency of seroma formation ranges from 15 to $18 \%$ after breast cancer surgery ${ }^{(4)}$. The pathogenesis of its formation is still not completely elucidated. Seroma can be developed by acute inflammatory exudates generated during the acute stage of wound healing in response to surgical trauma ${ }^{(5)}$.

The incidence of seroma was decreased by different methods, such as suturing the mastectomy flaps to the pectoralis muscle, immobilization of the ipsilateral arm in the early postoperative period, also using anti fibrinolytic agents such as tranexamic acid were found to be useful in minimizing fluid leakage therefore decreasing seroma formation ${ }^{(6)}$.

In this study, we have evaluated the effect of using gelfoam under mastectomy flaps to reduce seroma as the gelfoam is a widely used intraoperatively as gelatin compressed sponge for hemostasis. It is an organically degradable agent made of purified swine gelatin thought to help clot formation ${ }^{(7)}$.
Aim of the work was to assess the impact of using gelfoam in decreasing seroma formation following modified radical mastectomy.

\section{PATIENTS AND METHOD}

This study is a randomized controlled trial performed in Zagazig University Hospital between January 2019 and November 2020, involving eighty patients who had modified radical mastectomy and axillary clearance. Patients were randomly allocated into two groups; in each one forty patients. In group (A) we used the gelfoam sheets under mastectomy and axillary flaps, in group (B) we did the same surgical technique without using the gelfoam.

Inclusion criteria: Female patients with breast cancer stage II, average body weight and normal coagulation profile.

Exclusion criteria: Advanced breast cancer stage III and stage IV, patients who have a problem in blood clotting, history of long term use of steroids, uncontrolled diabetes or advanced liver disease and morbid obese patients.

\section{Procedure:}

The breast tissue was dissected and removed from medial to lateral. The axillary tail was continuously removed with axillary contents, then cleared from the lateral edge latissimus dorsi. Axillary vessels were identified by retracting pectoralis minor muscle. Dissection of axilla with preservation of the thoracodorsal bundle (nerve and vessels) (Figure 1) and 
preservation of the nerve to the serratus anterior (Figure 2) were carried out. The breast tissue and the axillary lymphatics were sent as one histopathological specimen. Hemostasis was secured with electrocautery; the operative area was irrigated with warm saline. In group (A) we inserted two sheets of gelfoam under the mastectomy flaps and one sheet under the axillary flap (Figure 3 and 4). We fixed the gelfoam sheets by two interrupted sutures (Vicryl 2/0) from the pectoralis major muscles passing through the gelfoam sheets to the subcutaneous tissue of the mastectomy flap (Figure 5). In group (B), the same procedures were done but without gelfoam use. Insertion of suction drain with two limbs, one placed under the upper flap and the other under the lower flap with its tips toward the axilla, then subcutaneous interrupted sutures were done and closure of the skin edges was performed with staples.

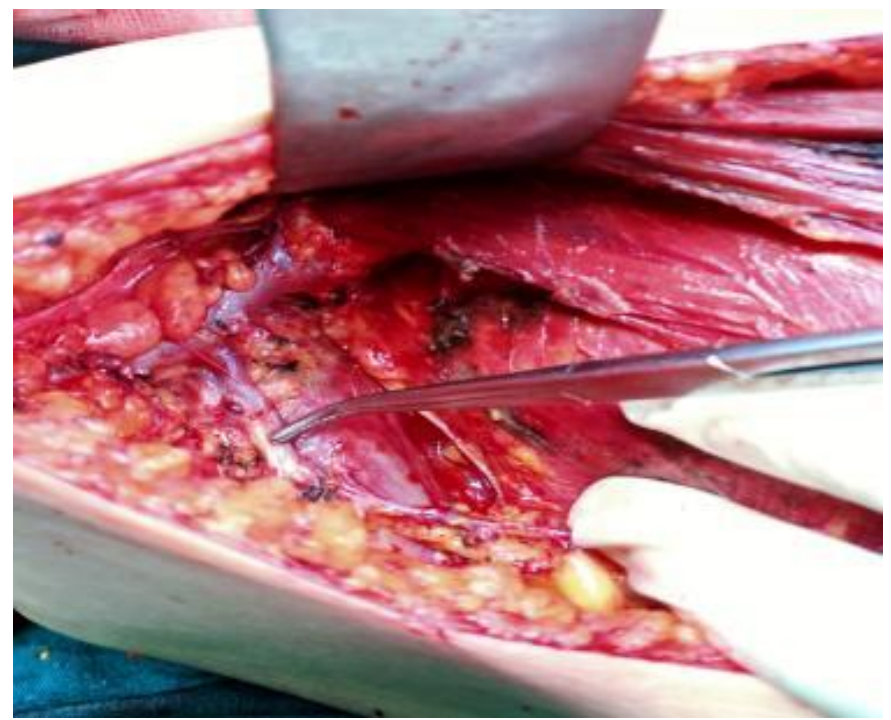

Figure (1): Preservation of the thoracodorsal bundle (nerve and vessels).

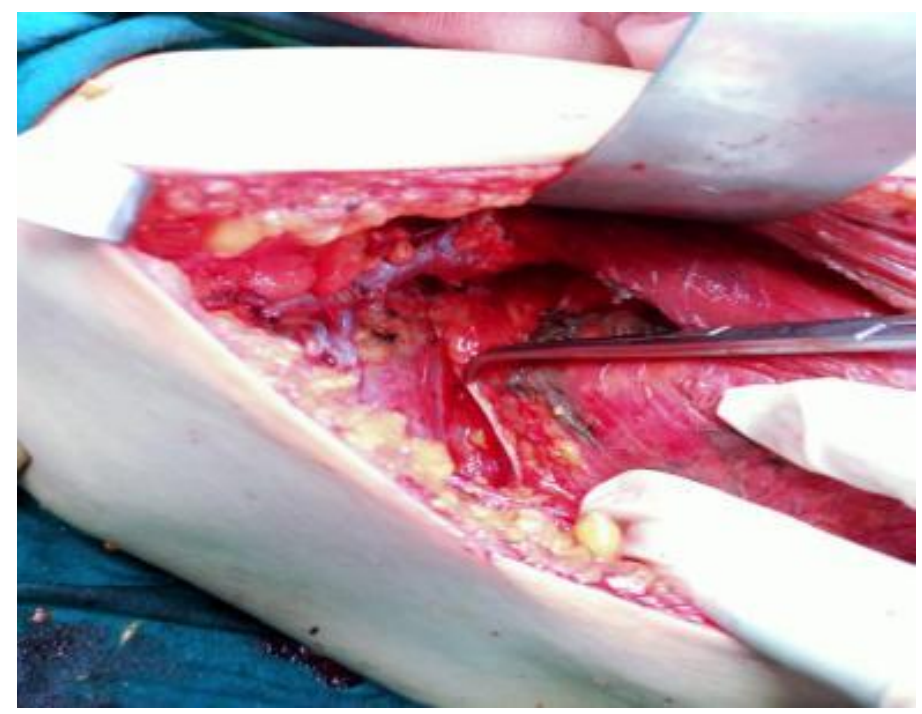

Figure (2): Preservation of the nerve to the serratus anterior.

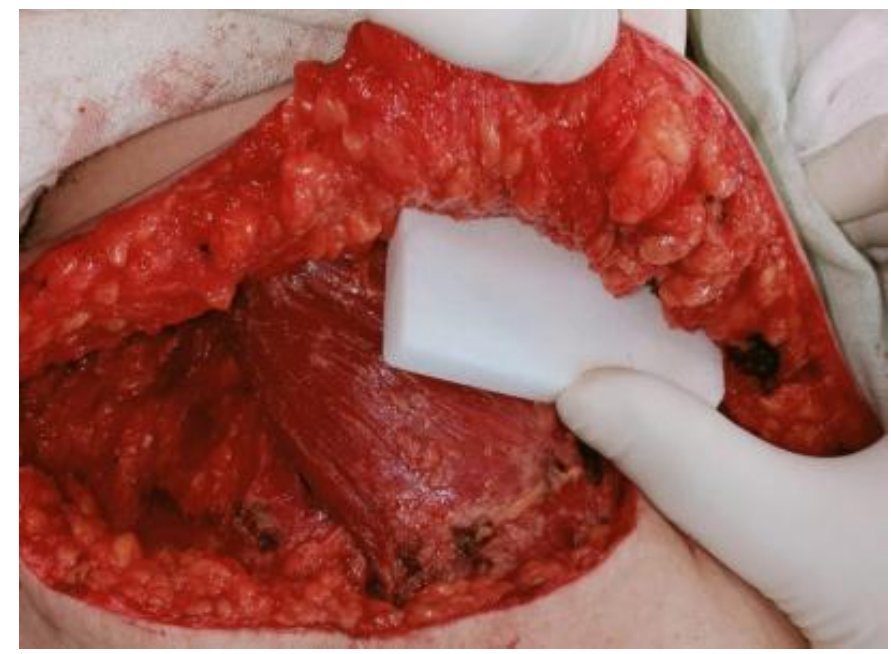

Figure (3): Inserting sheet of gelfoam under the upper mastectomy flap.

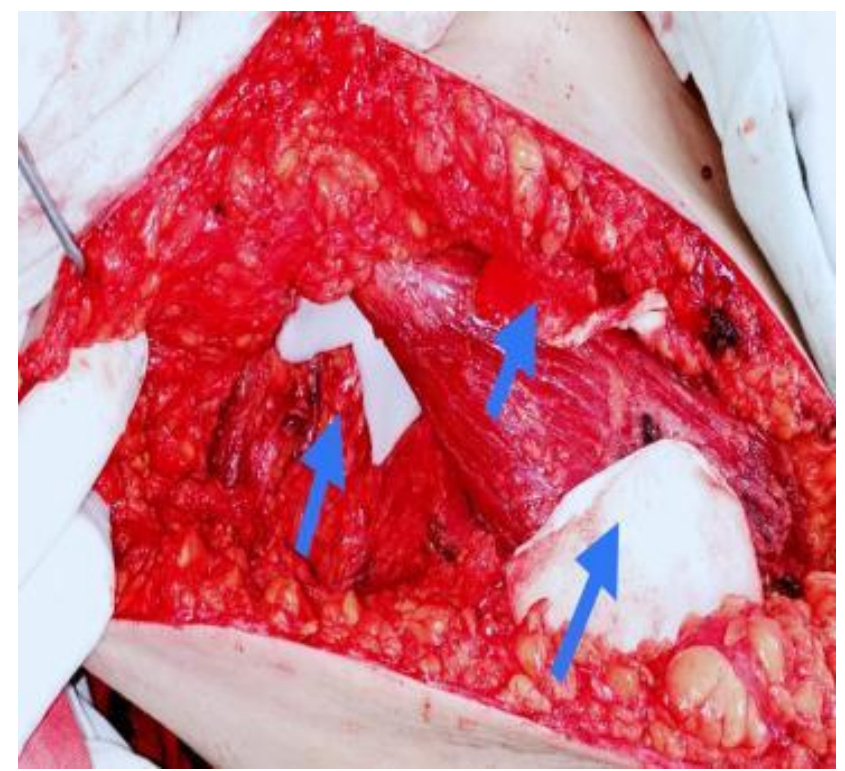

Figure (4): Sheets of gelfoam under the upper, lower and axillary flaps.

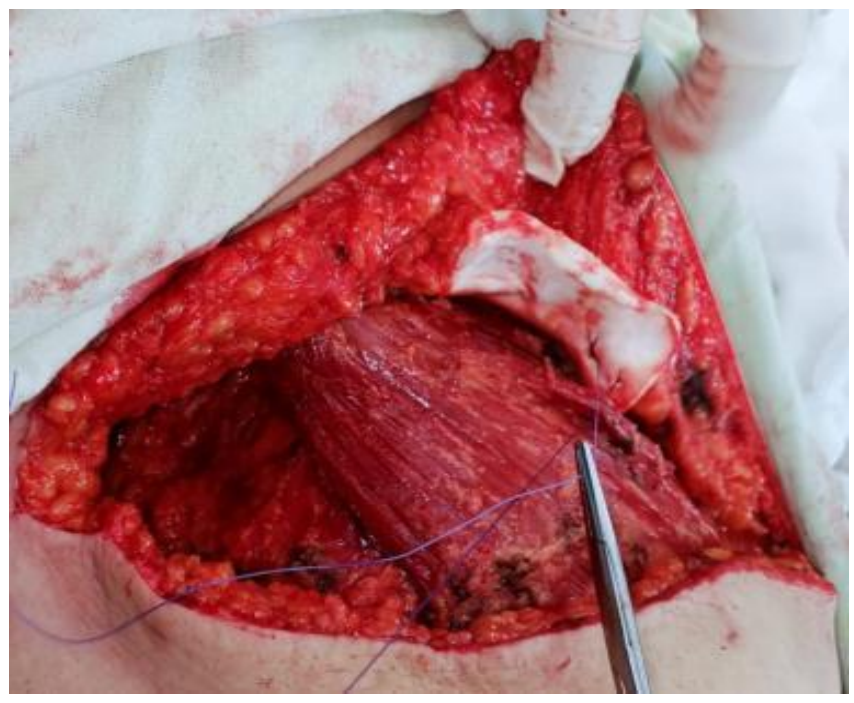

Figure (5): Fixing the gelfoam sheet by Vicryl 2/0 interrupted suture 


\section{Postoperative care:}

We removed the drain when the amount became less than $50 \mathrm{cc}$ in 24 hours for two days. Weekly followup for two months to detect seroma formation was done for all patients by clinical examination (redness and tenderness over the wound) and by superficial probe ultrasound to detect any collection. Any complication was recorded, sutures were removed 15 days postoperatively.

\section{Ethical approval and written informed consent:}

An approval of the study was obtained from Zagazig University Academic and Ethical Committee. Every patient signed an informed written consent for acceptance of the operation.

\section{Statistical analysis}

Data were verified, coded by the researcher and analyzed using IBM-SPSS for windows, version 23.0 (Copyright IBM Corp., Armonk, N.Y., USA. 2015). Descriptive statistics: Means, standard deviations (SD), and percentages were calculated.

Test of significances: Fisher exact test was used to compare the difference in distribution of frequencies among different groups. For continuous variables; independent t-test analysis was carried out for comparison. There was no specific calculation of the sample size. A significant p-value was considered when it is equal or less than 0.05 .

\section{RESULTS}

This table (1) shows that both groups were matched regarding age. As regard drain removal, patients of (group A) spent shorter duration with high statistically significant difference between both groups.

Table (1): General characteristics of the studied groups

\begin{tabular}{|c|c|c|c|c|}
\hline & $\begin{array}{c}\text { Group A } \\
\text { N=40 }\end{array}$ & $\begin{array}{c}\text { Group B } \\
\text { N=40 }\end{array}$ & t-test & P value \\
\hline $\begin{array}{c}\text { Age } \\
\text { Mean } \pm \text { SD }\end{array}$ & $45.5 \pm 9.35$ & $45.9 \pm 10.17$ & 0.18 & $\begin{array}{c}\text {.852 } \\
\text { NS }\end{array}$ \\
\hline $\begin{array}{c}\text { Days till drain removal } \\
\text { Mean } \pm \text { SD }\end{array}$ & $6.98 \pm 0.66$ & $14.8 \pm 1.95$ & 24.02 & $\begin{array}{c}<0.001 \\
\text { HS }\end{array}$ \\
\hline
\end{tabular}

NS: Non-significant difference HS: Highly significant difference

This table (2) shows decreased incidence of seroma formation among (group A) patients, with statistically significant difference between both of them.

Table (2): Seroma formation among both studied groups

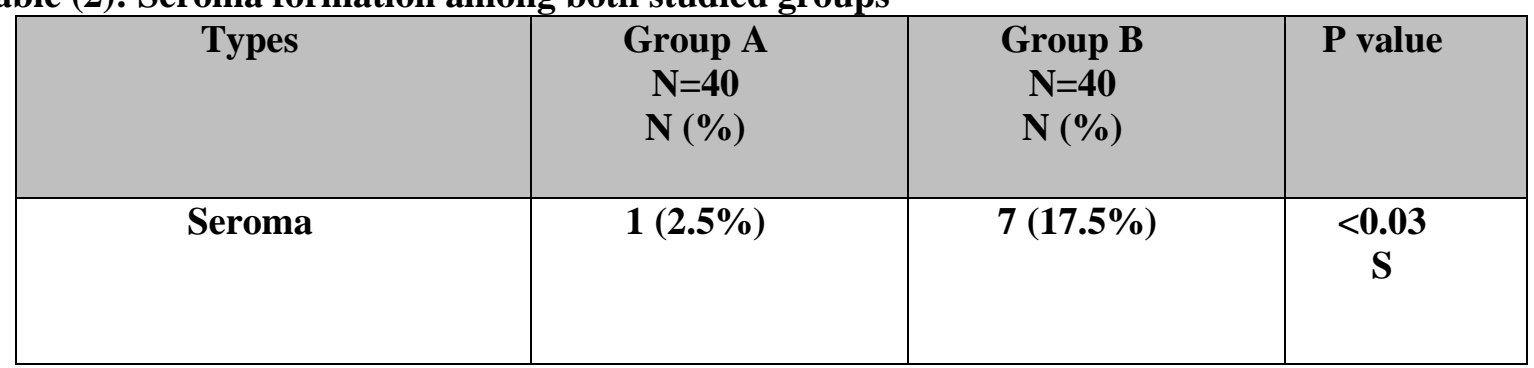

S: Significant difference 


\section{DISCUSSION}

Breast cancer is considered one of the most common leading causes of death for women ${ }^{(8)}$. In modified radical mastectomy, we remove the whole breast with overlying skin, areola, nipple, and most axillary lymph nodes ${ }^{(9)}$. The most frequent complication after mastectomy is seroma formation ${ }^{(\mathbf{1 0})}$. It is very important to be very cautious during breast surgery in order to minimize blood vessel and lymphatic leakage aiming to reduce appearance of seroma ${ }^{(\mathbf{1 1})}$.

In the present study, seroma formation was detected in 8 cases (out of 80 cases) with $10 \%$ of all cases.

Niranjan et al. ${ }^{(12)}$ performed a research on 46 patients underwent modified radical mastectomy in order to compare between the use of electrocautery and the use of harmonic scalpels, the incidence of seroma was 19\%.

In our study we proved that the use of gelfoam under mastectomy flaps during modified radical mastectomy leads to decrease seroma formation postoperatively as there was one case of seroma seen in 40 patients $(2.5 \%)$, while without gelfoam use, 7 cases of seroma appeared in 40 patients (17.5\%).

Porter et al. ${ }^{(13)}$ did study on 80 patients with breast cancer to compare between electrocautery and conventional scalpel usage in breast dissection, scalpel group was 38 patients and the electrocautery group was 42 patients. This study showed seroma formed in 16 wounds in electrocautery group compared to 5 in the scalpel group (38\% and $13 \%$, respectively; $\mathrm{P}=0.01$ ). Therefore, they proved that the use of traditional scalpel showed less seroma formation than electrocautery. In Anjani et al. ${ }^{\left({ }^{(14)}\right.}$ study 3 of 45 patients developed seroma with flap fixation and 15 of 35 patients without flap fixation developed seroma $(\mathrm{p}<0.001)$.

In our study patients of (group A) spent shorter duration with drain; about 7 days versus about 14 days among (group B) patients, with high statistically

significant difference between both of them. Similar observations were noted in the study done by Anjani $\boldsymbol{e t}$ al. ${ }^{(14)}$; the drain in patients with flap fixation was removed earlier than those without flap fixation $(18.61 \pm 1.24$ days in flap fixation group and $3.77 \pm 0.96$ days in group without flap fixation $\mathrm{p}<0.001$ ). So, we found that patients with short drain duration are less likely to develop seroma.

\section{CONCLUSION}

Using gelfoam during modified radical mastectomy is an effective technique that decreases the occurrence of seroma. Therefore we recommend this step at the end of mastectomy
Conflicts of interest: There are no interest conflicts.

Funding Acknowledgements: No financial support.

\section{REFERENCES}

1. Ibrahim A, Khaled H, Mikhail N et al. (2014): Cancer incidence in Egypt: results of national population-based cancer registry program. J Cancer Epidem., 14: 1-7.

2. Wooworth P, Mc Boyle M, Helmer S et al. (2000): Seroma formation after breast cancer surgery incidence a predicting factors. Am Surg., 66:444-450.

3. Sakkary M (2012): The value of mastectomy flap fixation in reducing fluid drainage and seroma formation in breast cancer patients. World J Surg Oncol., 10:8-13.

4. Akinci M, Cetin B, Aslans S et al. (2009): Factors affecting seroma formation after mastectomy with full axillary dissection. Acta Chir Belg., 109:481-483.

5. Ten Wolde B, Van den Wilenberg F, Keemers-Gels M et al. (2014): Quilting prevents seroma formation following breast cancer Surgery: closing the dead space by quilting prevents seroma following axillary lymph node dissection and mastectomy. Ann Surg Oncol., 21:802-807.

6. Zaidi S, Hinton C (2017): Breast cancer surgery without suction drainage and impact of mastectomy flap fixation in reducing seroma formation. European Journal of Surgical Oncology, 43(5): 32-37.

7. Lopez M, Rimm E (1998): Electrocautery usage disadvantages during mastectomy. Eur Surg Res., 30:148151.

8. Kuroi K, Shimozuma K, Taguchi $T$ et al. (2006): Evidence-based risk factors for seroma formation in breast surgery. Jpn J Clin Oncol., 36:197-206.

9. Loo W, Chow L (2007): Factors predicting seroma formation after mastectomy for Chinese breast cancer patients. Indian J Cancer, 44: 99-103.

10. Hoefer J, DuBois J, Ostrow L et al. (1990): Wound complications following modified radical mastectomy: an analysis of perioperative factors. The Journal of the American Osteopathic Association, 90(1): 47-53.

11. Kumar S, Lal B, Misra M (1995): Post-mastectomy seroma: a new look into the etiology of an old problem. Journal of the Royal College of Surgeons of Edinburgh, 40(5): 292-294.

12. Niranjan B, Anthana $S$ (2003): Comparative study of modified radical mastectomy using harmonic scalpel and electro cautery. Singapore Med J., 47:250-252.

13. Porter K, O'Conner S, Rimm E (1998): Electro cautery as a factor in seroma formation following mastectomy. Am J Surg., 176:8-11.

14. Anjani J, Amit O, Kuber S et al. (2016): Factors affecting seroma formation after modified radical mastectomy in patients of carcinoma breast: A prospective study. IJSS J Surg., 2(1):1-5. 